\title{
Matrizes para a Aquisição de Competências no Ensino de Urgências Clínicas / Ensino de Urgências Orientado por Competências
}

\section{PALAVRAS-CHAVE \\ - Educação Médica; \\ - Emergência; \\ - Competência Clínica; \\ - Educação Baseada em Competências; \\ - Aprendizagem.}

\section{KEYWORDS}

- Medical Education;

- Emergency;

- Clinical Competency;

- Competency-Based Education;

- Learning.

\section{Matrixes for Competency-Guided Teaching of Medical Emergency Care}

\author{
Maria Helena Senger \\ Maria Celeste Gonçalves Campos ${ }^{I}$
}

\section{RESUMO}

A Faculdade de Ciências Médicas e da Saúde (FCMS) da PUC-SP, campus Sorocaba, inseriu o aluno do internato em uma Unidade de Pronto Atendimento no estágio de urgências clínicas com os objetivos de melhorar o processo de ensino-aprendizagem em urgências durante a graduação e preparar o graduando para atuar em todos os níveis de atenção do Sistema Único de Saúde (SUS). Essa experiência levou à análise das competências a serem adquiridas para o atendimento ao paciente clínico nos serviços de urgência, considerando as especificidades desse tema. Para a aquisição de tais competências, foram construídas matrizes de conhecimentos, habilidades e atitudes, baseadas nas demandas por atendimento e nas oportunidades de aprendizagem, enfatizando que os conteúdos e as práticas devem ser oferecidos desde os anos iniciais da graduação. O modelo curricular da FCMS, desenvolvido por meio de módulos, mostrou ser propício a esta construção. Esse arcabouço pode ser estendido a escolas que usam outras estratégias pedagógicas e pode ser desenvolvido por várias disciplinas, conferindo ao ensino de urgências um caráter longitudinal e interdisciplinar.

\section{ABSTRACT}

The School of Medical Sciences and Health at the Pontifical Catholic University of São Paulo (PUC$-S P)$, Sorocaba campus, inserted its fifth and sixth year students into an Emergency Care Unit during their emergency care internship, aiming to enhance the teaching and learning of emergency care and to prepare them to perform at all care levels of the Unified Health System. This experience led to analysis of the competencies that must be acquired to assist clinical patients in urgent care, taking into account the specific characteristics of this matter. For the acquisition of these skills, matrixes of medical knowledge themes, abilities and attitudes were built, based on the patient's demands and on the learning opportunities, emphasizing that these contents and practices must be offered as from the beginning of the course. The school's curricular design, developed through modules, turned out to favor this process. This framework could be adopted by other schools that use different pedagogical strategies and even by other disciplines, bestowing on the teaching of emergency care a longitudinal and interdisciplinary characteristic.
Recebido em: 28/10/2014

Reencaminhado em: 21/01/2016

Aprovado em: 13/05/2016 


\section{ASSISTÊNCIA A URGÊNCIAS}

Os serviços de urgência constituem uma das áreas mais problemáticas do serviço público de saúde em função principalmente da demanda crescente por atendimento, motivada por vários fatores, entre os quais se destacam: alta morbimortalidade relacionada a violências e acidentes de trânsito até os 40 anos e, acima desta faixa etária, ligada às doenças do aparelho circulatório ${ }^{1}$; surtos de doenças infecciosas, em especial a dengue ${ }^{2}$; envelhecimento populacional, associado a maior prevalência de doenças crônicas e maior procura por serviços de saúde ${ }^{3}$; alta incidência de doenças respiratórias em função do tabagismo, poluição, condições ambientais inadequadas e baixo nível socioeconômico ${ }^{4}$.

O governo brasileiro tem elaborado políticas públicas que objetivam prover assistência adequada aos pacientes nos serviços de urgência. O ponto de partida foi a elaboração da Portaria 2.048, de novembro de 2002, que estabeleceu os princípios e diretrizes dos Sistemas Estaduais de Urgência e Emergência. Tal portaria continha elementos que foram posteriormente desenvolvidos, como as Centrais de Regulação Médica, normatizadas nessa portaria e que foram o embrião para a criação do atendimento Pré-Hospitalar Fixo (UPH ou UPA) e o Serviço de Atendimento Móvel de Urgência (Samu). As UPAs foram criadas com o objetivo de descentralizar o atendimento de pacientes com quadros agudos de média complexidade e diminuir a sobrecarga dos prontos-socorros dos hospitais de maior complexidade. Estes últimos, de acordo com o capítulo $\mathrm{V}$ da portaria citada, deveriam atuar como unidade de emergência referenciada ${ }^{5}$.

Em 2011, o Ministério da Saúde, com o objetivo de ampliar e qualificar o acesso humanizado e integral aos usuários em situação de urgência, de forma ágil e oportuna, instituiu a Rede de Atenção às Urgências no SUS, por meio da Portaria 1.600, de 7 de julho de 2011. A Rede é constituída pelos seguintes componentes, que deverão ser implantados até o ano de 2018: Promoção, Prevenção e Vigilância à Saúde; Atenção Básica em Saúde; Serviço de Atendimento Móvel de Urgência (Samu 192) e suas Centrais de Regulação Médica de Urgências; Sala de Estabilização; Força Nacional de Saúde; Unidades de Pronto Atendimento (UPA 24h) e o conjunto de Serviços 24 horas não hospitalares; Componente Hospitalar e Componente Atenção Domiciliar. Todas as portas de entrada do SUS devem se preparar para o atendimento inicial ao paciente em situação de urgência ${ }^{1}$.

\section{UNIDADES DE PRONTO ATENDIMENTO}

São estabelecimentos de complexidade intermediária entre as UBS (utilizando ou não a Estratégia da Saúde da Família) e a
Rede Hospitalar, criados com o objetivo de dar suporte à Atenção Básica e ao Samu e também reduzir o afluxo de usuários para as portas de entrada hospitalares ${ }^{6}$.

O Conselho Nacional de Secretários de Saúde (Conass) realizou, nos anos de 2012 e 2013, uma pesquisa para avaliar a implantação e o desempenho das UPAs na Rede de Atenção às Urgências, mediante entrevistas com gestores e profissionais dos serviços. Na época, o País contava com 162 unidades distribuídas em 19 estados. Foram pesquisadas 59 unidades distribuídas em seis estados, sendo que a maioria delas (19) estava localizada no Estado do Rio de Janeiro. Nas UPAs do Estado do Rio de Janeiro selecionadas para participar da pesquisa (19 das 42), o perfil dos usuários, de acordo com a opinião dos médicos, foi o seguinte: para o baixo risco, ocorreu predomínio de afecções do trato urinário e dor lombar/ afecções osteoarticulares (38\%), seguidos de infecções respiratórias (27\%); afecções do trato digestivo (25\%); hipertensão arterial (19\%); diabetes (17\%); e alcoolismo (16\%). No médio risco, houve predomínio de diabetes (34\%); doença pulmonar obstrutiva crônica (DPOC) e asma (32\%); infecções respiratórias agudas $(28 \%)$; e crise hipertensiva $(27 \%)$. A dor torácica foi o principal diagnóstico para o alto risco $(46 \%)$, seguido por DPOC e asma (24\%), hipertensão/crise hipertensiva (22\%) e violências e traumas $(15 \%)^{7}$.

Na cidade de Ribeirão Preto, no interior do Estado de São Paulo, as condições mais frequentes nesses serviços, de acordo com duas pesquisas, foram os quadros álgicos de várias etiologias, traumas e doenças respiratórias. Em ambas as pesquisas, a distribuição dos atendimentos de usuários adultos, segundo a faixa etária, mostrou predominância no grupo entre 20 e 40 $\operatorname{anos}^{8,9}$

\section{DEMANDA PELO TRABALHO}

Em serviços de urgência, a oferta de empregos é maior que a procura. Uma pesquisa realizada em 30 serviços de emergência hospitalar no Estado do Rio de Janeiro entre dezembro de 2005 e junho de 2006 mostrou que em $80 \%$ deles os postos de trabalho estavam incompletos ${ }^{10}$. Em junho de 2014, uma busca por empregos na área de Clínica Médica, no site do Sindicato dos Médicos do Estado de São Paulo, mostrou os empregos oferecidos apenas no dia $25 / 06 / 14$, com as seguintes ofertas na área de urgência: 34 vagas para trabalho em pronto-socorro e 19 vagas para trabalho em pronto atendimento. A experiência na área de urgências era exigida apenas em dez das vagas oferecidas para trabalho em pronto-socorro ${ }^{11}$.

O mercado é então propício, e o médico recém-formado acaba se inserindo precocemente nesses serviços. $\mathrm{O}$ relatório 
do Conass mostrou que, entre os médicos atuantes nas UPAs do Rio de Janeiro, 21,1\% haviam concluído a graduação havia menos de um ano e 34,2\% apresentavam menos de dois anos de formados?

Em pesquisa realizada com os egressos de 2011 do curso de Medicina da FCMS durante os meses de abril a junho de 2012, obtiveram-se as respostas de 50 alunos a um questionário enviado a todos os formandos. Trinta e dois estavam trabalhando em serviços de urgência, independentemente de estarem ou não cursando residência médica. Alguns deles referiram trabalhar em mais de um local, resultando em 38 postos de trabalho ocupados. As UPAs representaram o posto de trabalho mais frequentemente citado $(n=26 ; 68,4 \%$ dos 38 postos ocupados) $)^{12}$.

\section{ENSINO}

O aprendizado de urgências é inerente à formação médica, e a inserção do médico recém-formado nesse mercado reforça a necessidade de uma formação adequada durante a graduação. Isso tem sido motivo de preocupação das entidades representativas do ensino e da classe médica.

Em 2012, a Abem lançou o "Projeto Abem 50 anos - Dez anos das Diretrizes Curriculares Nacionais do Curso de Graduação em Medicina". Um dos propósitos desse projeto foi avaliar a o ensino de urgência nas escolas médicas. Foi observado que algumas escolas não têm matriz curricular de urgência; algumas universidades tradicionais não têm hospital com pronto-socorro para atendimento de urgência e emergência; há falta de professores e preceptores capacitados e com afinidade nessa área, bem como de integração e de um eixo longitudinal entre as diferentes áreas que atuam na urgência ${ }^{13}$.

As novas Diretrizes Curriculares Nacionais (DCN) do Curso de Graduação em Medicina recomendam que o aluno saiba identificar as situações de emergência desde o início do contato com pacientes e atuem de modo a preservar a saúde e a integridade física e mental das pessoas sob seus cuidados. Ainda, indicam a utilização de metodologias de ensino que privilegiem a participação ativa do aluno na construção do seu conhecimento ${ }^{14}$.

Nos Estados Unidos da América, a Medicina de Emergência é reconhecida como especialidade e existe interesse em padronizar o ensino. Com esse propósito, o American College of Emergency Physicians elaborou as suas diretrizes para o ensino de urgências com o intuito de ajudar as escolas no desenvolvimento de seus currículos. Trata-se de um modelo orientado por competências e enfoca a necessidade de oferecer os conteúdos desde os anos iniciais da graduação ${ }^{15}$.

\section{BREVE DESCRIÇÃO DO CURRÍCULO ATUAL DA FCMS - PUC-SP}

A Faculdade de Ciências Médicas e da Saúde (FCMS) de Sorocaba adotou, em 2006, um novo projeto pedagógico ${ }^{16}$. Nele, as disciplinas foram substituídas por módulos temáticos, desenvolvidos do primeiro ao quarto ano, usando metodologias ativas, centradas no estudante, com base nas seguintes atividades e estratégias de aprendizagem:

- Tutoria e estudo autodirigido: aprendizado a partir de problemas, baseados em situações do cotidiano, com tempo protegido para o estudo individual;

- Sustentações aplicadas: trabalho prático com experimentos, instrumentos e procedimentos;

- Sustentações teóricas: aula expositiva dialogada, mesas de discussão e debate, e reuniões anatomoclínicas;

- Habilidades: treinamento supervisionado para atingir competências fundamentais à prática médica sobre comunicação e aplicação da semiologia;

- Reflexão sobre a prática médica: atividade que busca um olhar integrador entre as facetas biológicas, éticas, legais, humanas, entre outras, presentes no treinamento prático;

- Prática em Atenção à Saúde (PAS): módulo longitudinal, desenvolvido do primeiro ao quarto ano, realizado em vários cenários da prática, priorizando a Atenção Básica e utilizando a problematização.

O internato (quinto e sexto anos) englobou a qualificação do processo de ensino-aprendizagem na área de urgências. Os alunos foram, então, inseridos no nível secundário da atenção às urgências, numa Unidade de Pronto Atendimento (UPA). Isso ocorreu em 2010, com os alunos do quinto ano, no estágio vinculado à Pediatria, e em 2011, com os alunos do sexto ano, no estágio na subárea de emergências clínicas, ligada à Clínica Médica.

O estágio na UPA é complementar ao que ocorre na Unidade Regional de Emergência (URE), também na área de Clínica Médica. A URE pertence ao nível terciário da atenção e recebe somente pacientes dos ambulatórios da instituição ou referenciados por intermédio de central de regulação médica e em situação de maior complexidade. As UPAs, por sua vez, trabalham com pacientes que procuram o serviço por demanda espontânea ou referenciados pela Atenção Básica. Têm como principais funções: prestar atendimento resolutivo e qualificado aos pacientes acometidos por quadros agudos ou crônicos agudizados de natureza clínica ou cirúrgica; trabalhar de forma articulada com os outros serviços do SUS, construindo fluxos coerentes e efetivos de referência e contrarreferência; realizar consulta médica em regime de pronto aten- 
dimento nos casos de menor gravidade ${ }^{6}$. Trata-se, portanto, de um cenário com grande volume de pacientes, permitindo ao aluno o contato com as doenças prevalentes na comunidade.

A inserção do aluno da FCMS nesse cenário levou à análise das competências a serem adquiridas para o atendimento ao paciente clínico nos serviços de urgência, considerando as especificidades do tema. As competências na área da saúde podem ser traduzidas como a capacidade de um ser humano cuidar do outro, colocando em ação conhecimentos, habilidades e atitudes necessários à prevenção e resolução de problemas de saúde em situações específicas do exercício profissional. Em outras palavras, é o resultado da aplicação combinada de pré-requisitos interligados, como habilidades cognitivas e práticas, conhecimento, motivação, orientação de valores, atitudes e emoções. Num modelo curricular orientado por competências, devem-se definir as competências esperadas em determinada experiência educacional e criar as condições de aprendizagem necessárias para alcançar os resultados desejados. É necessário, portanto, que os objetivos e as oportunidades de aprendizagem estejam alinhados ${ }^{17}$.

\section{OBJETIVOS}

Definir as competências a serem adquiridas para o atendimento ao doente clínico em serviços de urgência.

Construir matrizes de conhecimentos, habilidades e atitudes para aquisição das competências, para uso em diferentes modelos curriculares de cursos de Medicina.

\section{METODOLOGIA}

A pesquisa foi analisada e autorizada pelo Comitê de Ética e Pesquisa da FCMS (CAAE - Certificado de Apresentação para Apreciação Ética - 0115.0.154.000-11). Para a proposição das matrizes foram realizados três procedimentos que embasaram esta investigação.

Um buscou verificar os casos atendidos usualmente na UPA durante uma semana típica, de 29/08/2013 a 04/09/2013, sem feriados, escolhida aleatoriamente, com a finalidade de contemplar os temas mais recorrentes nas matrizes construídas. Os diagnósticos dos atendimentos dessa semana foram confrontados com os números anuais do ano anterior. Como houve grande semelhança, os dados daquela semana corresponderam aos utilizados porque foram submetidos à conferência dos diagnósticos pelas fichas de atendimento, não se baseando apenas no CID registrado pelos plantonistas.

Numa avaliação do estágio realizado na UPA sob a perspectiva dos alunos, já apresentada em publicação anterior, foi perguntado a eles que atendimentos lhes pareceram mais relevantes como contribuição à formação médica, além de pontos positivos e negativos do estágio, de forma aberta e ampla. As respostas dos estudantes contribuíram para a presente construção das matrizes ${ }^{18}$.

Por fim, foram identificados os temas relacionados à urgência oferecidos no ano de 2012 aos alunos do primeiro ao quarto ano da graduação, em levantamento no material disponibilizado via Modular Object-Oriented Dynamic Learning Environment (Moodle) da FCMS. Tal levantamento incluiu todas as atividades pedagógicas ofertadas aos alunos: os problemas usados nas sessões de tutoria e os temas das sustentações aplicadas e teóricas e das habilidades. Estes temas foram destacados nas matrizes.

Os três procedimentos foram associados e comparados às referências bibliográficas sobre o tema, nacionais e internacionais. Tais referências foram utilizadas na execução do presente trabalho, para definição final das competências a serem adquiridas durante o estágio de urgências clínicas, considerando os níveis secundário e terciário da atenção à saúde $\mathrm{e}^{14,15,19-23}$.

Assim, procedeu-se à construção de matrizes de conhecimentos, habilidades e atitudes a serem desenvolvidos durante toda a graduação, usando diferentes estratégias de ensino-aprendizagem oferecidas no currículo do curso. As estratégias receberam as denominações utilizadas no projeto pedagógico de Sorocaba, mas também foram adequadas a atividades didáticas correspondentes, visando a sua adaptação a outros currículos: (a) tutoria; (b) sustentações aplicadas / aulas práticas em laboratório (morfofuncional); (c) sustentações teóricas / aulas teóricas; (d) habilidades / aulas práticas em ambulatórios, enfermarias e pronto-socorro; (e) habilidades / aulas práticas em laboratório de simulação; (f) Prática em Atenção à Saúde / Saúde Coletiva / Atenção Básica; (g) reflexão sobre a prática médica / Bioética; (h) internato / ambulatório e enfermaria; (i) internato/ pronto-socorro; (j) internato/Samu; (k) internato / UPA; (l) internato / UTI; (m) discussão de caso clínico; (n) artigos de revista; (o) seminários; (p) eletiva; (q) e-learning; (r) estudo autodirigido.

\section{RESULTADOS}

A Tabela 1 apresenta os dados dos atendimentos realizados na UPA no período de 29/08/2013 a 04/09/201 e durante o ano de 2012, em ordem de frequência até a décima colocação. Observa-se certa semelhança entre os diagnósticos mais frequentes em ambos os levantamentos.

Os atendimentos que os alunos consideraram mais relevantes para sua formação durante o estágio na UPA foram: demanda por atestados para faltar ao trabalho; dor torácica; lombalgia; doenças respiratórias; cólica nefrítica; asma; doenças infecciosas (dengue, malária, etc.); outros (cefaleia, diarreia, unha encravada, etc.); dor abdominal; pacientes da 


\begin{tabular}{|c|c|c|c|c|c|}
\hline \multicolumn{6}{|c|}{$\begin{array}{l}\text { TABELA } 1 \\
\text { ealizados nos períodos especificados. }\end{array}$} \\
\hline \multicolumn{3}{|c|}{$29 / 08 / 2013$ a $04 / 09 / 2013$} & \multicolumn{3}{|c|}{ Ano de 2012} \\
\hline $1^{\mathrm{o}}$ & IVAS & $9,4 \%$ & $1^{\circ}$ & Doenças do aparelho respiratório & $28,3 \%$ \\
\hline $2^{\mathrm{o}}$ & Gripe & $7,8 \%$ & $2^{\mathrm{o}}$ & Sintomas, sinais e achados anormais & $19,5 \%$ \\
\hline $3^{o}$ & Traumas menores & $7,3 \%$ & $3^{\circ}$ & Doenças osteomusculares & $8,1 \%$ \\
\hline $4^{\mathrm{o}}$ & Lombalgia & $6,2 \%$ & $4^{\mathrm{o}}$ & Lesões, envenenamentos e causas externas & $8,0 \%$ \\
\hline $5^{\circ}$ & Amigdalite & $5,0 \%$ & $5^{\circ}$ & Doenças do aparelho digestivo & $7,7 \%$ \\
\hline $6^{\circ}$ & Sinusite & $5,0 \%$ & $6^{\circ}$ & Doenças infecciosas e parasitárias & $5,4 \%$ \\
\hline $7^{\circ}$ & ITU & $4,5 \%$ & $7^{\circ}$ & Doenças do aparelho geniturinário & $3,6 \%$ \\
\hline $8^{\circ}$ & Dor Abdominal & $3,8 \%$ & $8^{\circ}$ & Não informado & $3,5 \%$ \\
\hline $9^{\circ}$ & Cefaleia & $3,5 \%$ & $9^{\circ}$ & Doenças do aparelho circulatório & $3,3 \%$ \\
\hline $10^{\circ}$ & Asma & $3,0 \%$ & $10^{\circ}$ & Fatores que influenciam no estado de saúde & $3,0 \%$ \\
\hline
\end{tabular}

Atenção Básica; idosos; causas externas de morbimortalidade (mordeduras por animais, traumas, etc.); transtornos mentais; situações de urgência (AVC, arritmia, IRA, diabetes descompensado e HDB); infecção do trato urinário. Entre os pontos positivos, os alunos destacaram a possibilidade de trabalhar o receituário médico, e os pontos negativos se relacionaram à curta duração do estágio ${ }^{18}$.
No levantamento no material disponibilizado via Moodle da FCMS identificaram-se oportunidades para o aprendizado de urgências desde os anos iniciais da graduação. Tais oportunidades estão identificadas nas matrizes. Os dados culminaram com a descrição de 18 competências a serem adquiridas no ensino de urgências clínicas durante a graduação médica (Quadro 1).

\section{$\begin{array}{lll}1 & \\ & \text { QUADRO } 1\end{array}$ \\ Competências a serem desenvolvidas pelo processo de ensino-aprendizagem de urgências clínicas durante a graduação médica.}

Atuar no atendimento ao paciente vítima de parada cardiorrespiratória.

Reconhecer situações de risco iminente à vida e instalar medidas de sustentação à vida.

Atuar nos principais agravos à saúde em serviços de urgência.

Compreender o conceito de triagem com classificação de risco.

Realizar história objetiva e exame físico dirigido.

Fazer o diagnóstico diferencial e priorizar o diagnóstico provável baseado na apresentação do paciente.

Prescrever medicamentos mais comuns em serviços de urgência.

Reconhecer as indicações, custos e evidências dos exames diagnósticos mais comuns em serviços de urgência.

Realizar o adequado encaminhamento dos pacientes que necessitem de cuidados em outros níveis de atenção à saúde.

Atuar nos surtos de doenças infecciosas de acordo com os protocolos do Ministério da Saúde e do Serviço de Vigilância Epidemiológica do município.

Reconhecer as necessidades individuais de todos os usuários e as de grupos específicos (ex.: idosos, trabalhadores).

Elaborar um plano de alta que inclua medidas de promoção e prevenção de doenças.

Fazer prescrição de medicamentos para uso domiciliar.

Referenciar para os programas da Atenção Básica quando necessário.

Estabelecer uma boa relação médico-paciente.

Documentar, notificar e manter anotações clínicas apropriadas e legíveis.

Respeitar e obter o respeito em relação ao trabalho em equipe.

Gerenciar efetivamente o tempo disponível para atendimento do usuário. 


\section{CONSTRUÇÃO DAS MATRIZES}

Para a aquisição das competências foram construídas as matrizes de conhecimentos, habilidades e atitudes, apresentadas nos Quadros de 2 a 5. Sua elaboração deu-se da seguinte forma, respeitando os anos da graduação: conteúdos, modalidades didáticas aplicáveis, possíveis cenários para a aprendizagem e disciplinas ou áreas de conhecimento que, mesmo não estando inseridas no ambiente da urgência, podem contribuir no processo ensino-aprendizagem por representarem interface com aquele conteúdo. As oportunidades de aprendizado encontradas no material disponível no Moodle foram identificadas com asterisco.

O Quadro 2 apresenta os conhecimentos gerais necessários à aquisição das competências na atuação em urgências. Levaram-se em consideração algumas situações mais comuns no nível secundário da atenção (como a prática da referência e contrarreferência) e também a opinião dos alunos (conforme a pesquisa anterior, que apontou os atendimentos a idosos e pacientes da Atenção Básica, além do preenchimento recorrente de atestados como marcantes para os estudantes) $)^{6,18}$.

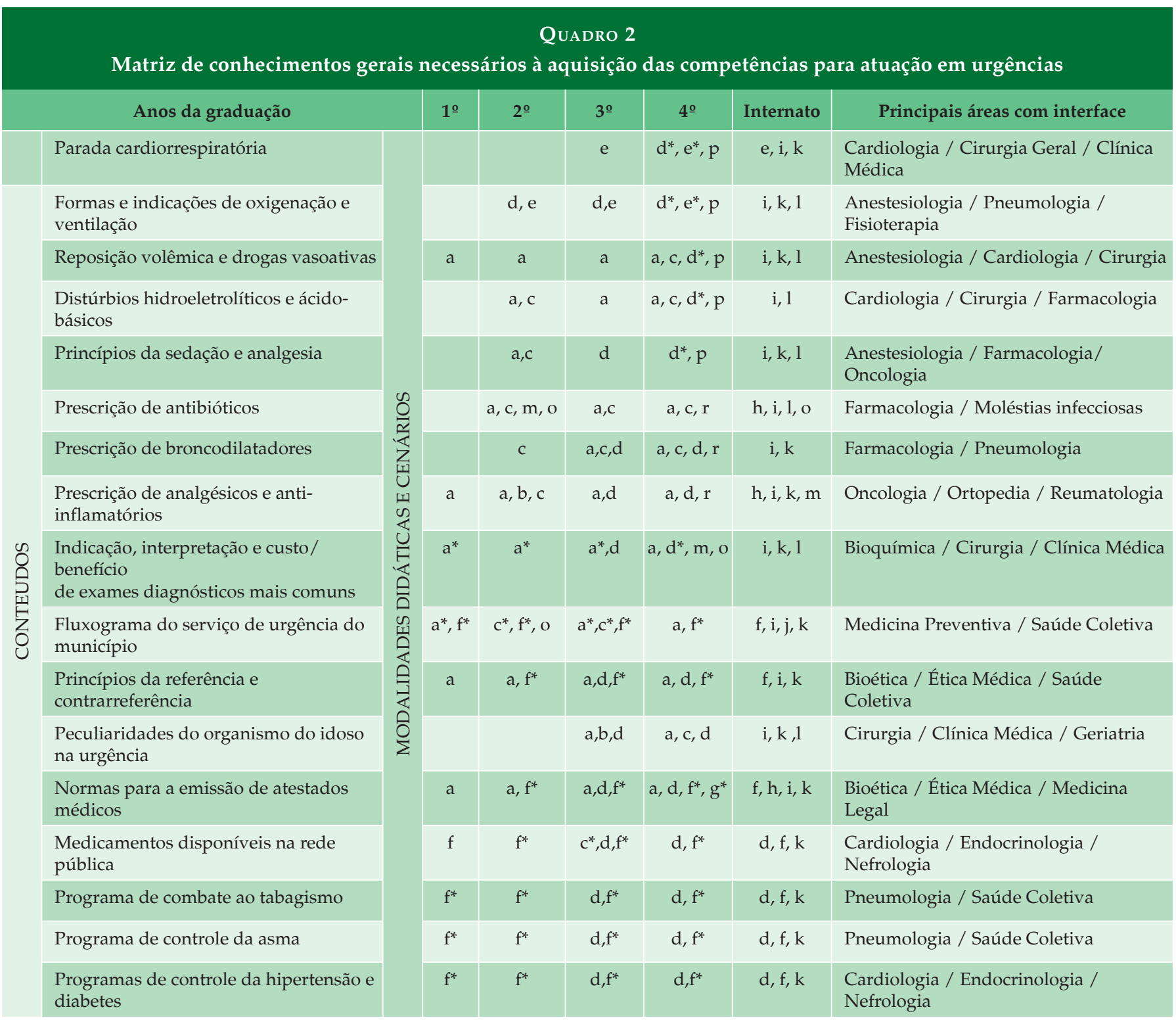

*Oportunidades de aprendizagem identificadas no Moodle, utilizadas para os alunos do primeiro ao quarto ano em 2012.

(a) tutoria; (b) sustentações aplicadas / aulas práticas em laboratório (morfofuncional); (c) sustentações teóricas / aulas teóricas; (d) habilidades / aulas práticas em ambulatórios, enfermarias e pronto socorro; (e) habilidades / aulas práticas em laboratórios de simulação; (f): Prática em Atenção à Saúde / Saúde Coletiva / Atenção Básica; ( $g$ ) reflexão sobre a

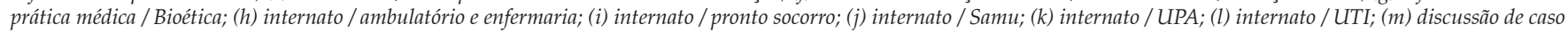
clínico; (n) artigos de revista; (o) seminários; ( $p$ ) eletiva; $(q)$ e-learning; $(r)$ estudo autodirigido. 


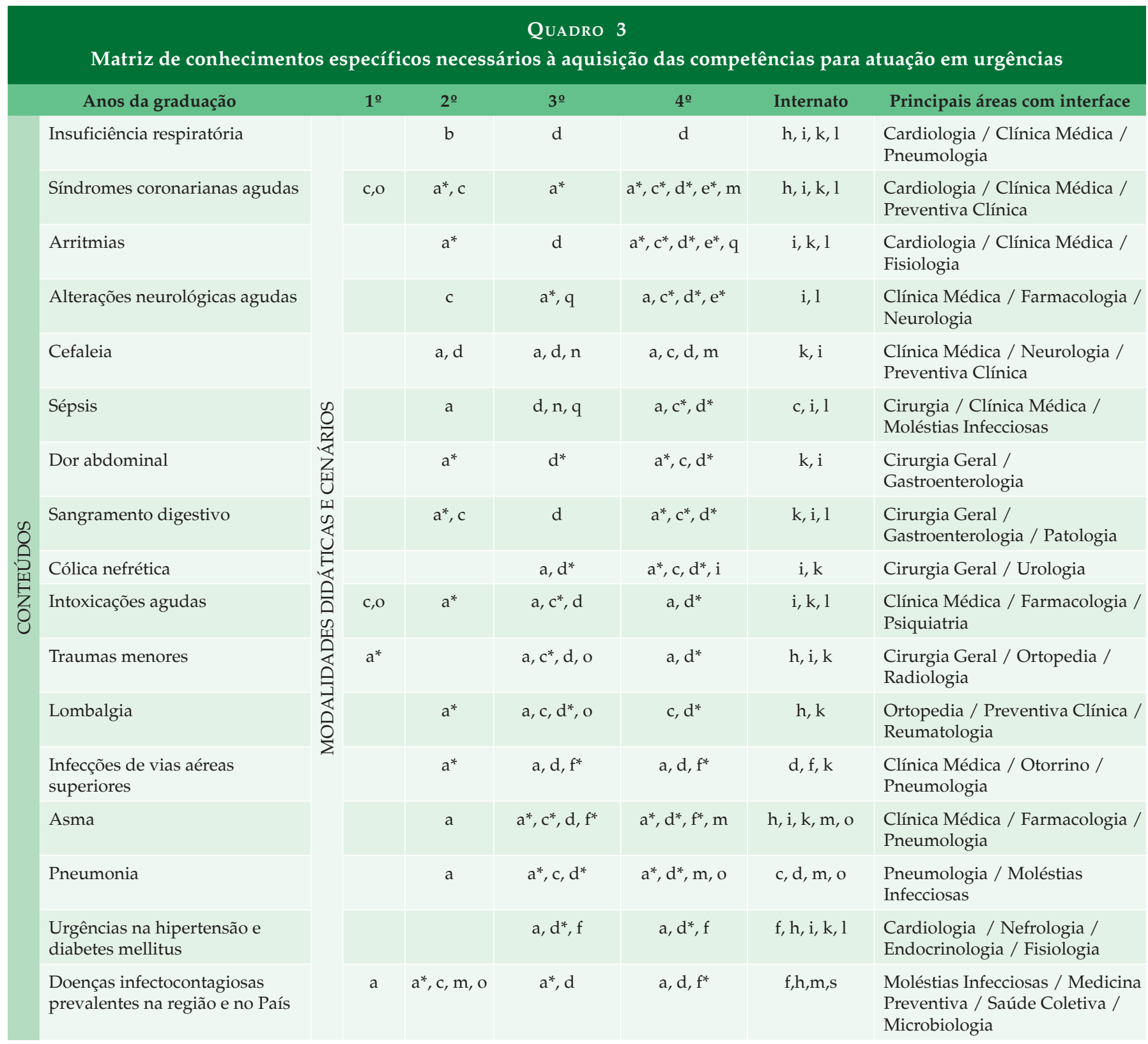

*Oportunidades de aprendizagem identificadas no Moodle, utilizadas para os alunos do primeiro ao quarto ano em 2012.

(a) tutoria; (b) sustentações aplicadas / aulas práticas em laboratório (morfofuncional); (c) sustentações teóricas / aulas teóricas; (d) habilidades / aulas práticas em ambulatórios, enfermarias e pronto socorro; (e) habilidades / aulas práticas em laboratórios de simulação; (f): Prática em Atenção à Saúde / Saúde Coletiva / Atenção Básica; ( $g$ ) reflexão sobre a

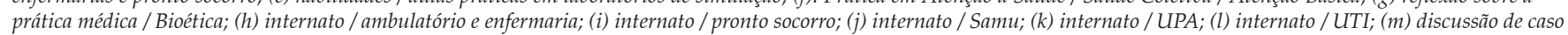
clínico; ( $n$ ) artigos de revista; (o) seminários; ( $p$ ) eletiva; ( $q$ ) e-learning; $(r)$ estudo autodirigido.

Da mesma forma, o Quadro 3 apresenta os conhecimentos específicos. A escolha das patologias privilegiou as situações clínicas potencialmente emergenciais em qualquer cenário, as urgências relacionadas às doenças crônicas não transmissíveis prevalentes no País (DM, HAS), as condições prevalentes no cenário da Atenção Secundária (quadro álgicos, doenças respiratórias e traumas menores) e os agravos infecciosos ${ }^{3,8,9,24}$.
Já o Quadro 4 apresenta as habilidades necessárias à aquisição das competências na atuação em urgências ${ }^{21,22,23}$.

E, finalmente, o Quadro 5 mostra algumas das atitudes importantes para a atuação em urgências, considerando as DCN e algumas referências internacionais ${ }^{14,21,22,23}$. 


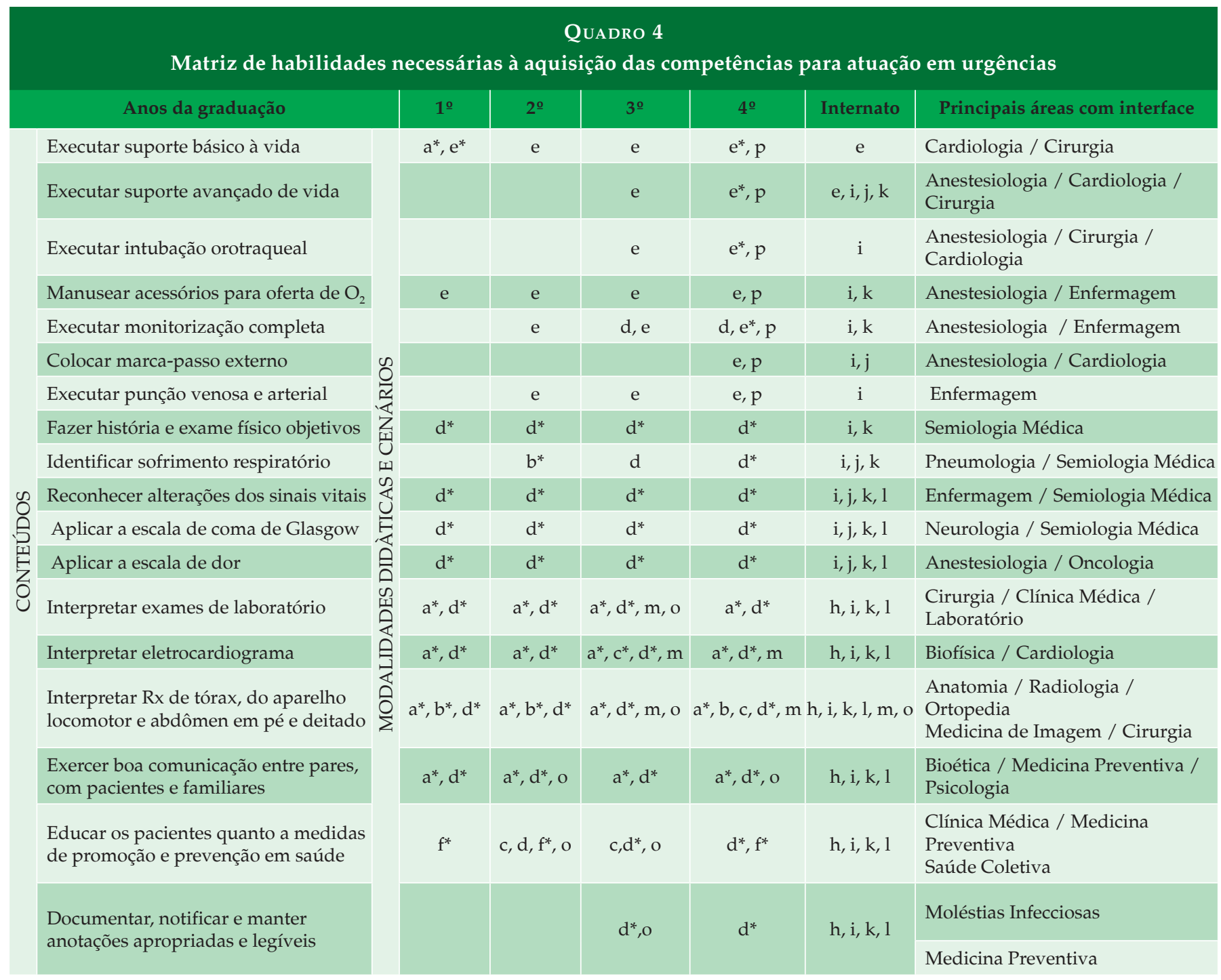

*Oportunidades de aprendizagem identificadas no Moodle, utilizadas para os alunos do primeiro ao quarto ano em 2012.

(a) tutoria; (b) sustentações aplicadas / aulas práticas em laboratório (morfofuncional); (c) sustentações teóricas / aulas teóricas; (d) habilidades / aulas práticas em ambulatórios,

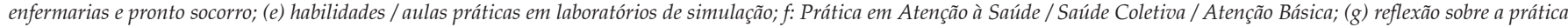

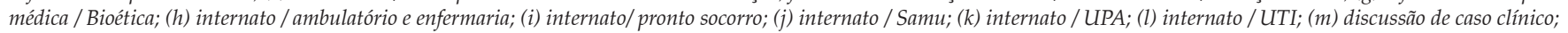
(n) artigos de revista; (o) seminários; ( $p$ ) eletiva; ( $q$ ) e-learning; $(r)$ estudo autodirigido.

\section{DISCUSSÃO}

Os resultados do presente trabalho mostram uma possibilidade concreta de utilização de matrizes de competências, voltadas para a aprendizagem de urgências clínicas em adultos, com a proposição de longitudinalidade deste tema no currículo médico. Ainda, a construção destas matrizes levou em conta sua aplicabilidade em diferentes propostas de projetos pedagógicos. A opção por um modelo de ensino orientado por competências encontrou respaldo em algumas das referências bibliográficas usadas na execução do trabalho ${ }^{14,19-23}$.
As sugestões para o ensino de urgências clínicas consideraram o nível secundário da atenção à saúde, cenário reconhecido com base nos dados de atendimentos da UPA, a impressão dos alunos sobre o estágio e também as possíveis oportunidades para o aprendizado de urgências desde o início do curso. Este desenho longitudinal permite que a apropriação das competências ocorra de forma recorrente, com aproximações sucessivas.

A análise dos dados dos atendimentos mostrou semelhança com aqueles pesquisados em outras localidades e confir- 
mou a alta incidência de doenças respiratórias, juntamente com os quadros álgicos de variadas etiologias, em especial os de causa osteomuscular e também os pequenos traumas ${ }^{7,8,9}$.

A opinião dos alunos trouxe à tona algumas situações frequentes no nível secundário da atenção às urgências, como a presença do idoso, pacientes com dificuldades de atendimento ou desgarrados da Atenção Básica, demanda por atestados para justificar faltas ao trabalho e a possibilidade de trabalhar o receituário médico ${ }^{18}$.

Com relação a estas opiniões, a demanda de atendimento dos idosos se consolida com base em outros dados, pois o Brasil deverá se tornar, no corrente ano (2015), o sexto país do mundo em número de idosos (estimativa de 32 milhões). Este fato se associa a maior prevalência de doenças crônicas e maior procura por serviços de saúde, incluindo os de urgência ${ }^{25}$. Quanto aos pacientes da Atenção Básica, estes também são frequentes em serviços de urgência e acredita-se que isso não se deva apenas à falta de vagas para consulta na Atenção Básica ${ }^{10}$. Nessas situações, cabe ao médico não só atender com também fazer os encaminhamentos ou contrarreferenciamentos necessários. Finalmente, o preenchimento de receitas e/ou atestados é um procedimento que ocorre em 50\% dos casos atendidos em pronto-socorro não referenciado, aspecto usualmente negligenciado em muitos currículos ${ }^{26}$. Portanto, tais tópicos merecem ser trabalhados no currículo médico das urgências.

O estudo no Moodle mostrou que algumas oportunidades para o ensino de urgência ocorrem desde os anos iniciais da graduação. Houve tutorias relacionadas à síndrome coronariana aguda em todos os anos a partir do segundo ano. Por outro lado, o treinamento para o manuseio de acessórios para oferta de oxigênio foi oferecido apenas no quarto ano. Trata-se de um procedimento simples e de suma importância no ambiente da urgência. Então, as oportunidades que já existem merecem ser mantidas ou mesmo incentivadas e outras necessitam ser criadas, o que vale para qualquer projeto pedagógico. Mas, para isso, é preciso instituir o controle do que vem sendo aplicado pelos professores.

$\mathrm{Na}$ construção das matrizes de conhecimentos gerais e específicos, buscou-se o preparo do aluno para atendimento ao doente potencialmente grave e ao portador de doenças prevalentes no meio onde vive, temas que precisam ser contemplados no currículo. Nos Estados Unidos da América, a Academic Emergency Medicine sugere dez situações às quais o aluno deve ser exposto no estágio de urgências - dor abdominal, estado mental alterado, parada cardíaca, dor no peito, sangramento gastrointestinal, dor de cabeça, envenenamento, desconforto respiratório, choque e trauma -, a maioria das quais está representada nas matrizes ${ }^{22}$.
No Canadá, a Canadian Association of Emergency Physicians Undergraduate Education Committee publicou, em 2013, um modelo de currículo para ser aplicado no estágio de urgências, baseado em competências, tendo como pano de fundo os CanMeds (modelo curricular para o ensino médico no Canadá, baseado em competências e desenvolvido pelo Royal College of Physicians and Surgeons of Canada) ${ }^{27}$. Também são citados 40 tópicos importantes para o aprendizado dos alunos e há semelhanças entre os mais frequentes - dor torácica, falta de ar, alteração do nível de consciência, anafilaxia / reação alérgica grave, dor abdominal, perda de consciência (síncope), choque, convulsão, parada cardiorrespiratória, dor de cabeça, trauma menor - e aqueles referidos nas matrizes ${ }^{23}$. Ou seja, houve confluência dos conteúdos levantados no presente trabalho com os dados de duas importantes instituições.

A identificação e a sugestão de oportunidades de aprendizagem desde os anos iniciais da graduação apontam o eixo longitudinal como norteador do ensino de urgências e seguem as recomendações da Abem e de referências internacionais ${ }^{15,20,21,28,29}$. O desenho longitudinal permite aproximações sucessivas do mesmo objeto, utilizando variadas metodologias de ensino e em diferentes cenários de aprendizagem ${ }^{16}$. Mesmo em discussões de casos clínicos ou nas visitas aos pacientes na enfermaria, o debate pode ser ampliado para se considerar a apresentação inicial destes na sala de emergência, num exercício do raciocínio clínico ${ }^{20,22}$. Ou seja, mesmo não estando em ambiente da urgência, potencialmente todos os professores e quase todas as áreas de conhecimentos podem contribuir neste processo, incluindo as chamadas "básicas". As disciplinas ou departamentos devem perceber que, juntos, poderão abordar um assunto de forma mais efetiva e compartilhada, com ganho no aprendizado do aluno ${ }^{30}$. Considera-se também o aprendizado interprofissional ao se sugerir, por exemplo a participação da Fisioterapia no ensino da ventilação mecânica.

O treinamento em laboratórios de simulação também é essencial para a aquisição das habilidades e deve ser iniciado no primeiro ano, com o suporte básico de vida ${ }^{13,20,21}$. Outras habilidades de fácil entendimento, como o manuseio de acessórios para a oferta de oxigênio, também podem ser apreendidas precocemente. Obviamente, é imprescindível o treinamento nos cenários de prática, de preferência no ambiente da urgência, também desde os anos iniciais da graduação. $\mathrm{O}$ aluno pode, acompanhado do professor, executar anamnese e exame físico em casos de menor complexidade e até participar do atendimento aos pacientes que são vítimas de parada cardiorrespiratória, nos anos subsequentes ${ }^{20,22}$. Recomenda-se também que as equipes que atuam na urgência (médicos, enfermeiros e fisioterapeutas, entre outros) sejam convidadas a 
contribuir com o ensino, quando possível ${ }^{25}$. Uma pesquisa realizada com 104 escolas americanas mostrou que o serviço de emergência está disponível para o primeiro ano em 25 escolas (23,4\%), para o segundo ano em $22(20,6 \%)$, para o terceiro ano em $15(14,0 \%)$ e para quarto em $42(39,3 \%)$ escolas $^{29}$.

Também é chamada a atenção para as disciplinas básicas, como a Biofísica, no ensino do eletrocardiograma, e é reforçado o aprendizado interprofissional com a participação da enfermagem no manuseio de acessórios para ventilação, acesso venoso e arterial. Já a matriz de atitudes aponta aquelas mais importantes no ambiente da urgência, mas que na realidade podem ser adquiridas em todos os cenários da prática ${ }^{13,22}$.

\section{CONCLUSÃO}

As competências apresentadas e a construção das matrizes tiveram o intuito de apresentar ideias para o processo ensino-aprendizagem em urgências. Não têm caráter prescritivo, portanto. Uma planilha simples, nas mãos de pessoas envolvidas com o ensino de urgências, pode se transformar numa matriz ao ser completada segundo as ideias aqui propostas. É imperioso, no entanto, que se aponte para a longitudinalidade do ensino das urgências, obedecendo a graus crescentes de complexidade.

\section{REFERÊNCIAS}

1. Brasil. Ministério da Saúde. Portaria no 1.600 de 07 de julho de 2011. Reformula a Política Nacional de Atenção às Urgências e institui a Rede de Atenção às Urgências no Sistema Único de Saúde (SUS). Diário Oficial da União. Brasília, 08 jul. 2011; p. 69.

2. Monitoramento dos casos de dengue e febre de chikungunya até a Semana Epidemiológica 26, 2015. Bol Epidemiol [on line]. 2015;46(20) [capturado 22 set. 2015]. Disponível em: http://portalsaude.saude.gov.br/images/ pdf/2015/julho/27/2015-027---Dengue-SE-26--.pdf

3. Pazin Filho A, Scarpelini A. Medicina de Emergência na FMRP-USP: Você pode fugir, mas não há como se esconder! Medicina (Ribeirão Preto). 2010;43(4):432-43.

4. Fonseca FR, Vasconcelos CH. Estudo da distribuição de doenças respiratórias no estado de Santa Catarina, Brasil. Cad Saúde Coletiva. 2011;19(4):454-60.

5. Brasil. Ministério da Saúde. Portaria 2.048 de 05 de novembro de 2002. Normatiza e estabelece diretrizes dos Sistemas Estaduais de Urgência e Emergência. Diário Oficial da União. Brasília, 12 nov. 2002; Seção 1, p. 32-54.

6. Brasil. Ministério da Saúde. Portaria $n^{\circ} 1.601$ de 07 de julho de 2011. Estabelece as diretrizes para a implantação do componente Unidades de Pronto Atendimento (UPA 24h) e o conjunto de serviços de urgência 24 horas da Rede de Atenção às Urgências, em conformidade com a Política Nacional de Atenção às Urgências. Diário Oficial da União. Brasília, 08 jul. 2011; Seção 1, p. 70-72.

7. Brasil. Conselho Nacional de Secretários de Saúde. Rede de Atenção às Urgências e Emergências: avaliação da implantação e do desempenho das Unidades de Pronto Atendimento (UPAs). - Brasília (DF): CONASS; 2015. 400 p. (CONASS Documenta, 28).

8. Souza Gomide MF, Pinto IC, Gomide DMP, Zacharias FCM. Perfil de usuários em um serviço de pronto atendimento. Medicina (Ribeirão Preto). 2012;45(1):31-8.

9. Garcia VM, Reis RK. Perfil de usuários atendidos em uma unidade não hospitalar de urgência. Rev Bras Enferm. 2014;67(2):261-7.

10. O'Dwyer GO, Oliveira SP, Horsth MH. Avaliação dos serviços hospitalares de emergência do programa QualiSUS. Ciênc Saúde Coletiva. 2009;14(5):1881-90.

11. Sindicato dos Médicos de São Paulo. Banco de Empregos Médicos [on line]. São Paulo: SIMESP; 2014 [capturado 28 jun. 2014]. Disponível em: www.simesp.com.br.

12. Campos MCG, Senger MH. O trabalho do médico recém-formado em serviços de urgência. Rev Soc Bras Clín Méd. 2013;11(4):355-9.

13. Fraga GP, Pereira Junior, GA, Fontes CER. O ensino de urgência e emergência nos cursos de graduação de medicina: situação atual e recomendações para a matriz curricular. In: Lampert JB, Bicudo AM, organizadores. 10 anos das Diretrizes Curriculares Nacionais dos cursos de graduação em Medicina. Rio de Janeiro: Associação Brasileira de Educação Médica; 2014. p.41-56.

14. Brasil. Ministério da Educação. Conselho Nacional de Educação. Câmara de Educação Superior. Resolução nº 03 de 20 de junho de 2014. Institui as Diretrizes Curriculares Nacionais do Curso de Graduação em Medicina e dá outras providências. Diário Oficial da União. Brasília, 23 jun. 2014; Seção 1, p. 8-11.

15. Manthey DE, Burns GD, Jobe KA, Kessler CS, Harkin KE, Binder L, et al. ACEP's Academic Affairs Committee Members Guidelines for Undergraduate Education in Emergency Medicine [on line]. New York: ACEP; 2008 [capturado 22 set. 2015]. Disponível em: http:/ /www.acep.org/ content.aspx?id=29866.

16. Pontifícia Universidade Católica de São Paulo. Projeto Pedagógico (PP) do Curso de Medicina da Faculdade de Ciências Médicas de Sorocaba do Centro de Ciências Médicas e Biológicas (CCMB) PUC-SP. São Paulo: PUC-SP; 2010. Aprovado pela reitoria em 01/12/10. 
17. Bollela VR, Machado JL. Internato baseado em competências. Belo Horizonte: MedVance; 2010. (Coleção educação para as profissões da saúde).

18. Campos MCG, Senger MH. Avaliação do estágio de urgências clínicas em uma unidade de pronto atendimento sob a perspectiva dos alunos. Rev Bras Educ Méd 2014;38(1):103-12.

19. Gontijo ED, Alvim C, Megale L, Melo JRC, Lima MECC. Matriz de competências essenciais para a formação e avaliação de desempenho de estudantes de medicina. Rev Bras Educ Méd. 2013;37(4):526-39.

20. Coates WC. An educator's guide to teaching emergency medicine to medical students. Acad Emerg Med. 2004;11(3):300-6.

21. Hobgood C, Anantharaman V, Bandiera G. International Federation for Emergency Medicine model curriculum for medical student education in emergency medicine. Int J Emerg Med. 2010;3(1):1-7.

22. Manthey DE, Ander DS, Gordon DC, Morrissey T, Sherman SC, Smith MD, et al; Clerkship Directors in Emergency Medicine (CDEM) Curriculum Revision Group. Emergency medicine clerkship curriculum: an update and revision. Acad Emerg Med. 2010;17(6):638-43.

23. Penciner R, Woods RA, McEwen J, Lee R, Langhan T, Bandiera G. Core competencies for emergency medicine clerkships: results of a Canadian consensus initiative. CJEM. 2013;15(1):24-33.

24. Martins HS, Brandão Neto RA, Scalabrini Neto A, Velasco IT. Emergências clínicas: abordagem prática. $7^{\mathfrak{a}}$ ed. Barueri (SP): Manole; 2012.

25. Serbim AK. Redes de apoio social percebido por idosos usuários de um serviço de emergência. Porto Alegre; 2012. Mestrado [Dissertação] - Escola de Enfermagem da Universidade Federal do Rio Grande do Sul.

26. Pazin-Filho A, Borges MC, Scarpelini S. A Inserção na comunidade do eixo longitudinal de emergências da Faculdade de Medicina de Ribeirão Preto da Universidade De São Paulo (FMRP-USP). In: Bollela VR, Germani AC, Cam- pos HH, Amaral EM, organizadores. Educação baseada na comunidade para as profissões da saúde: aprendendo com a experiência brasileira. Ribeirão Preto: FUNPEC-Editora; 2014. p.149-59.

27. Frank JR, editor. The CanMEDS 2005 physician competency framework. Better standards. Better physicians [on line]. Better care. Ottawa: The Royal College of Physicians and Surgeons of Canada; 2005 [capturado 22 set. 2015]. Disponível em: http://www.royalcollege.ca/portal/ page/portal/rc/common/documents/canmeds/resources/publications/framework_full_e.pdf

28. Beckers SK, Timmermann A, Muller MP, Angstwurm M, Walcher F. Undegraduate medical education in emergency medical care: a nationwide survey at German medical schools. BMC Emerg Med. 2009; 9:7.

29. Khandelwal S, Way DP, Wald DA, Fisher J, Ander DS, Thibodeau L, Manthey DE. State of undergraduate education in emergency medicine: a National Survey of Clerkship Directors. Acad Emerg Med. 2014;20(1):92-5.

30. Harden RM. The integration ladder; a tool for curriculum planning and evaluation. Med Educ. 2000;34:551-7.

\section{CONTRIBUIÇÃO DOS AUTORES}

Maria Helena Senger e Maria Celeste Gonçalves Campos participaram de todas as etapas da pesquisa e da elaboração do artigo.

\section{CONFLITO DE INTERESSES}

Os autores declaram não haver conflito de interesses.

\section{ENDEREÇO PARA CORRESPONDÊNCIA}

Maria Helena Senger

Faculdade de Ciências Médicas e da Saúde de Sorocaba - PUC-SP

Rua Joubert Wey, 290

Vergueiro - Sorocaba

CEP: 18030-070 SP

E-mail: mhsenger@pucsp.br 